\title{
Heat- and humid-proof adhesive joints for optical pickup
}

\author{
Manabu OCHI*, Yasuo KITADA** and Yoshiro KONISHI** \\ *Hitachi, Ltd., Research \& Development Group \\ 832-2 Horiguchi, Hitachinaka, Ibaraki 312-0034, Japan \\ E-mail: manabu.ochi.ub@hitachi.com \\ **Hitachi-LG Data Storage Inc. \\ 3-22-23 Kaigan, Minato-ku, Tokyo 108-0022, Japan
}

Received: 1 April 2019; Revised: 19 June 2019; Accepted: 25 June 2019

\begin{abstract}
Our previously proposed method for arranging adhesive joints for an optical assembly was adopted to resist long-term exposure to severe environments. This method is expected to passively compensate for permanent optical misalignment due to long-term exposure to hot and humid conditions by arranging the bonding direction and position of each optical component in order to tend to displace or tilt a desired amount in a desired direction over time. We calculated the beam spot deviation on a detector, which is an indicator of misalignment of the optical assembly due to heat, humidity, and time effects on adhesive joints. We arranged to adhesively bond the laser diode to the holder from the top so that the laser diode could be lifted up due to the moisture expansion of the adhesive joint over time. The developed adhesive joints arrangement had a great advantage in that upward displacement of the laser diode compensates for beam spot deviation caused by tilt of the prism and displacement of the detector along the warp deformation of the housing. The calculated and measured results showed that the beam spot deviation was within about $\pm 5 \%$ after the optical pickup was left at $60^{\circ} \mathrm{C}$ and $90 \% \mathrm{RH}$ for 336 hours. It is concluded that our method for arranging adhesive joints of each component effectively compensates for passively permanent optical misalignment that occurs after long-term exposure to hot and humid conditions.
\end{abstract}

Keywords : Optical disc drive, Positioning, Viscoelasticity, Moisture expansion, Outgassing shrinkage, Adhesive

\section{Introduction}

Optical discs, such as compact discs (CDs), digital versatile discs (DVDs), and Blu-ray discs (BDs), are most commonly used for distributing music, movies, and other data because of their removability, non-contact readability and writability, and ease of use. An optical disc drive uses an optical pickup to read data from and write data into a disc. A typical read and write operation involves keeping the beam spots on the target data track of the rotating disc and converting the reflected beams into servo signals and information data. This can be done with an optical assembly including a laser diode, photo detector, and objective lens. The laser beams from the laser diode are reflected and transmitted to optical components and are then focused on a data track on a record layer of the disc by the objective lens. The reflected beams subsequently return to the pickup and are directed to the detector. Slight misalignment of the optical components results in the position deviation of the beam spot on the detector. This position deviation is incorrectly considered as servo signals, decreasing the precision of beam spot position with respect to the data track on the record layer of the disc. The pickup is, therefore, required to maintain a precise optical alignment and a long-term stability in order to have highly reliable reading and writing operations.

Adhesive joints are employed to fix lenses, mirrors, and other components made of metal and plastic to a housing (Sakamoto and Saito, 2014). This is because an adhesive joint can be cured within seconds at room temperate during the assembly alignment process and, in short, is suitable for high volume production. In addition, adhesive joints with low elastic modulus are selected for lenses and mirrors to prevent them straining due to cure shrinkage and the thermal expansion difference between bonded components and housing material (Chikuma, 1998; Matuo, 1998; Yanagihara, 
2001). These adhesive joints, however, are affected by various environmental factors like temperature and humidity (Iida, 2007; Prabhu et al., 2007). Permanent dimensional changes in adhesive joints generally occur due to moisture absorption, outgassing, and residual stress, resulting in instability in the optical assembly alignment (Iwai, 2001).

To solve this problem, several types of optical integrated laser diodes, which are single-packaged with a photodetector, are applied in pickups (Yoshida et al., 2000; Nemoto and Honda, 2002; Manoh et al., 2003), especially, for automotive use. They show excellent environmental resistance due to the adjacent placement of the laser chip and the detector. However, they require an extremely high-precision semiconductor manufacturing process to mount multiple chips and optical elements with accurate relative positions between each other in a single small package, which is costly and limits the production amount (Kato et al., 2001; Miyauchi et al., 2003; Terashi et al., 2003). This paper focuses on a pickup using a discrete optical assembly consisting of individual optical and electronic components. This type of pickup allows optical configurations for servo signal detection to be flexible enough to be compatible with all disc formats. It can also ease the introduction of high-power laser diodes supporting fast writing operation and adopt cheaper components. Yet long-term optical alignment stability is also needed.

To compensate for misalignment of optical assembly, several studies have proposed methods for correcting servo signal offset due to optical misalignment over time (Yoshimoto et al., 1990; Nakai et al., 2013). Those methods involve subtracting additional values, such as an average servo signal level during track jump and an imbalance in light intensity on the detector, from the servo signal itself. However, these methods have several limitations. They have difficulty correcting short-term effects due to temperature changes in operation. In addition, they have a limitation in the correction amount or allowable misalignment value. Thus, adhesive joints for a discrete pickup need to be designed so as to resist long-term exposure to severe environments while maintaining optical alignment. Nevertheless, we have no way to completely suppress the amount of displacement and tilt of all optical components, as long as optical components are bonded by adhesive joints with low elastic modulus.

This study was an attempt to passively compensate for the permanent optical misalignment that occurs after longterm exposure of an optical pickup to a temperature of $60^{\circ} \mathrm{C}$ and a relative humidity (RH) of $90 \%$. To do that, we adopt our previously proposed method that can passively compensate for the reversible optical misalignment due to temperature change. This method is to arrange the bond position and direction of each component in order to tend to displace or tilt a desired amount in a desired direction with temperature variations (Ochi et al., 2006, 2008). This method allows the effects of displacement and tilt of each component on the optical misalignment to balance each other out.

In this paper, the effectiveness of this method against the permanent optical misalignment was tested through linear viscoelastic structural calculations and acceleration experiments. We first calculated the beam spot deviation on the detector, which is an indicator of misalignment of the optical assembly over time due to effects of heat and humidity on the adhesive joints. Specifically, we performed linear viscoelastic structural calculations under thermal loading with Abaqus ${ }^{\circledR}$. For the calculation, we defined the viscoelastic behavior of the adhesive by a Prony series approximation of a relaxation modulus master curve, modeled by the generalized Maxwell, and an Arrhenius plot of the time-temperature shift factor. We also included the dimensional change due to moisture absorption or outgassing shrinkage of the adhesive joint in the coefficient of thermal expansion, which varies over time. After the calculations, we identified the primary cause of the deviation and compared two types of bonding structures for the laser diode. We then developed the two prototype optical pickups and evaluated them through an acceleration experiment. Finally, our method of arranging adhesive joints for an optical assembly was proved to also effectively enhance long-term optical alignment stability.

\section{Optical pickup}

\subsection{Configuration}

Figure 1 shows an optical pickup for a slim-type BD drive, which can read and write BDs, DVDs, and CDs. This pickup includes two sets of optical assembly. One is for compatibility with DVDs and CDs, and the other is only for BDs. It also uses mechanical parts such as a two-axis actuator with two objective lenses for respective disc formats. They are adhesively bonded to a metal die-cast housing and are covered with a metal plate, which provides electromagnetic interference (EMI) shielding and heat spreading.

The main target of this research is a discrete optical assembly compatibility with DVDs and CDs as shown in Figure 2. A laser diode, grating, prism, lens 1, mirror, objective lens, lens 2, and detector are well aligned in the optical paths. A laser beam, emitted from the laser diode, is first divided into three diffracted beams, 0 th and \pm 1 st order, by the grating to obtain the tracking servo signal. The three beams are focused on a data layer of the disc by the objective lens after 
reflecting and transmitting the prism, lens 1 , and mirror. The beams reflected from the disc return to the prism and then pass through it. The beams are finally detected by the detector through the lens 2 with astigmatic aberrations increased to obtain the focusing servo signal.

Sensing areas on the detector are divided into either two or four segments. This enables servo signals to be obtained by measuring the output current of each segment with the change in the intensity distributions of the beam spots. Because the output current of each segment also depends on the beam spot position, the position deviation of the 0th order diffracted beam spot on the detector is inspected and quality controlled as an indicator of optical misalignment. The position deviation signals in the horizontal and vertical directions, $P D_{H}$ and $P D_{V}$ respectively shown in Figure 2, are given by

$$
\begin{aligned}
& P D_{H}=\frac{\left(I_{A}+I_{B}\right)-\left(I_{C}+I_{D}\right)}{I_{A}+I_{B}+I_{C}+I_{D}} \times 100 \\
& P D_{V}=\frac{\left(I_{A}+I_{D}\right)-\left(I_{B}+I_{C}\right)}{I_{A}+I_{B}+I_{C}+I_{D}} \times 100
\end{aligned}
$$

where $I_{A}, I_{B}, I_{C}$, and $I_{D}$ are the output current measured on segments A, B, C, and D, respectively.

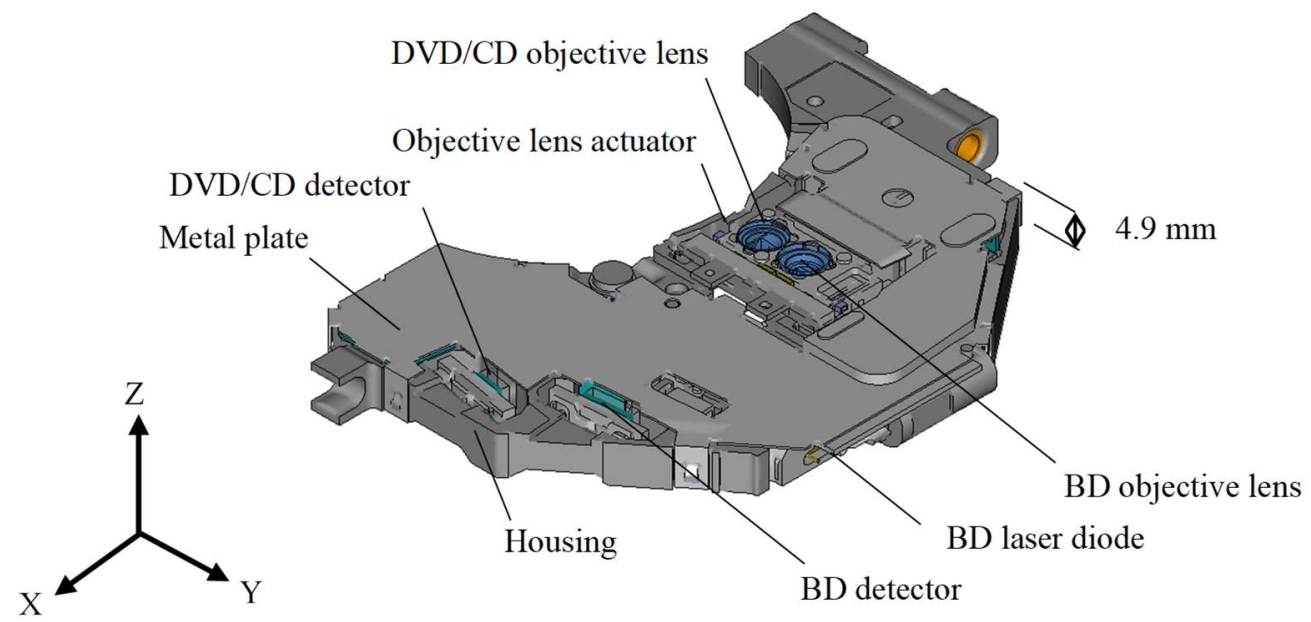

Fig. 1 Appearance view of optical pickup for slim-type BD drive, which can read and write BDs, DVDs, and CDs. This pickup is comprised of two optical assemblies: one is for compatibility with DVDs and CDs, and the other is only for BDs.

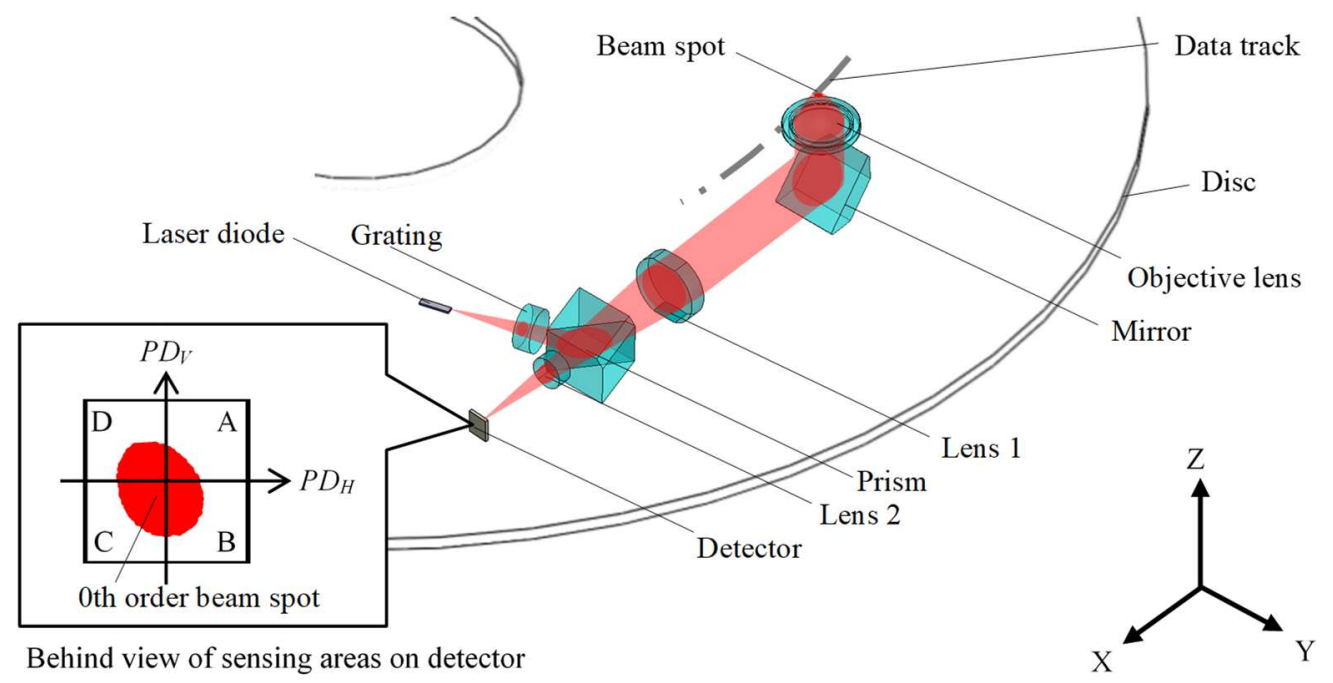

Fig. 2 Configuration of discrete optical assembly for compatibility with DVDs and CDs, and arrangement of sensing areas on detector. 


\subsection{Adhesive joints}

Figure 3 shows adhesive joints fixing the optical assembly and objective lens actuator to the housing. Several kinds of adhesive are used depending on the components. Adhesive A is thermal cured, Adhesive G is moisture cured, and the others are ultraviolet (UV) cured.

Figure 4 shows bonding directions of major contributing optical components. Each laser diode and detector is handled by being bonded on its holder for heat dissipation. After being precisely positioned, they are adhesively bonded to the housing in their front-back directions basically. There are several reasons for this. First, these adhesive joints are commonly a few mm thick to give them sufficient travel margins in positioning, so they expand and shrink in the thickness directions with temperature variations. In addition, their slight displacements in either the vertical or horizontal direction correspond to the significant deviation of the beam spot on the detector. For the same reason, the prism is bonded in the direction perpendicular to the normal to its reflecting surface.

Adhesive joints both between the laser diode and its holder and between the laser diode and the metal plate are, however, restricted to be bonded in the vertical direction sufficiently to conduct heat through a large area. On one hand, the adhesive bonding between the holder and the metal plate (Adhesive $\mathrm{G}$ ) has a small influence on displacement of the laser diode because of its low elastic modulus. On the other hand, the adhesive bonding the laser diode to its holder (Adhesive A) may be especially sensitive to humidity because of its high moisture expansion, which will be discussed in more detail below, though Adhesive A is very thin (at most several dozen $\mu \mathrm{m}$ thick) and its coefficient of thermal expansion is not very high. We simultaneously had a strong expectation that the beam spot deviation on the detector in the vertical direction could be balanced easily by arranging either of two bonding directions of the laser diode: one is to bond the laser diode down to the top of the holder (hereinafter, referred to as bottom-bonded) as shown in Figure 4(a) and the other is to bond the laser diode up to the bottom of the holder (hereinafter, referred to as top-bonded) as shown in Figure 4(b). We expected this to be very effective, since these arrangements should not affect any other optical component behaviors. We then tested both of them. Prototypes with the bottom-bonded laser diode were prepared by rewiring cables back-to-front between the flexible printed circuit and the pins of the laser diode, after turning the holder with the laser diode upside-down from the prototypes with the top-bonded laser diode.

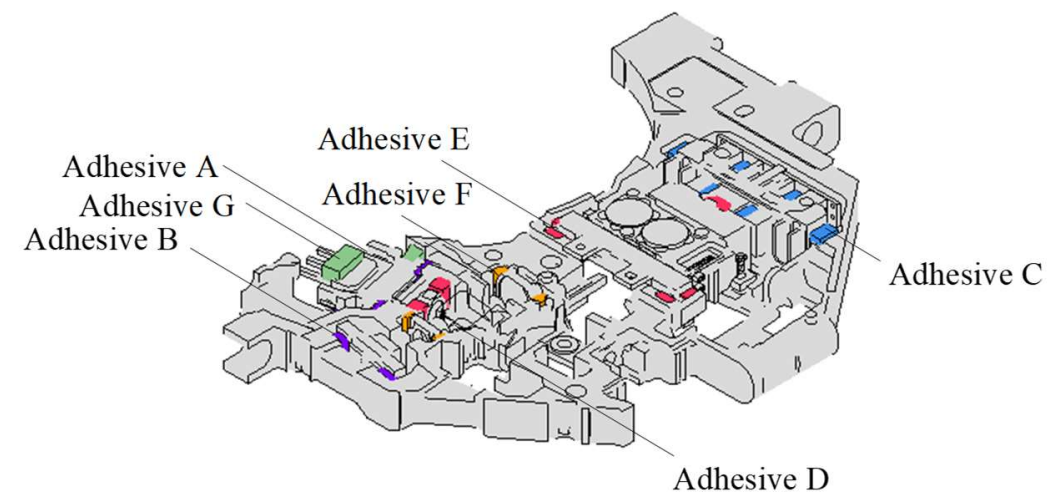

Fig. 3 Adhesive joints regarding an optical assembly and objective lens actuator. They are color-coded by kind of adhesive.

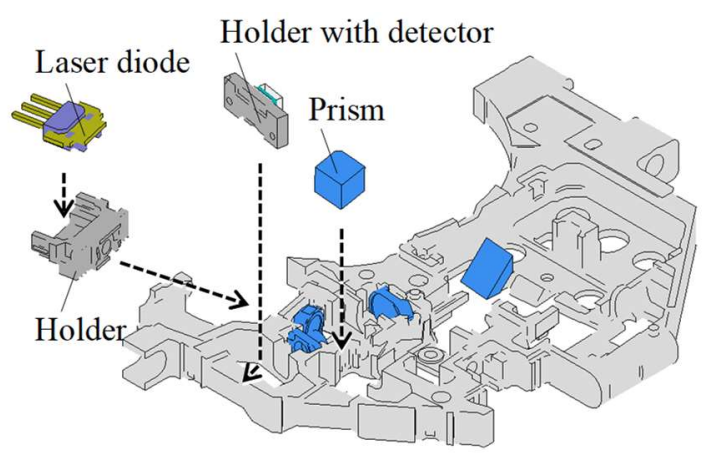

(a) Bottom-bonded

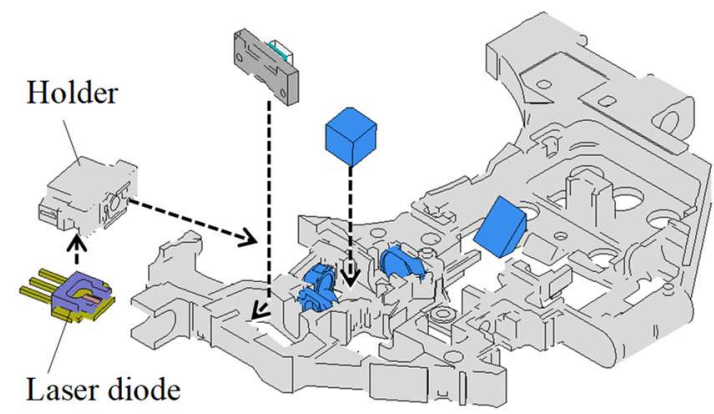

(b) Top-bonded

Fig. 4 Bonding directions of major contributing optical components. Prism and holders of laser diode and detector are bonded to housing in each arrow direction. (a) and (b) show different bonding directions between laser diode and holder. 


\section{Formulation of position deviation of beam spot on detector}

The position deviation of the beam spot on the detector becomes the sum of two components, as shown in equation (3): $\Delta_{1}$ is due to the displacements and tilts with respect to their optical axes of the optical components, and $\Delta_{2}$ is due to their axial displacements. $\Delta_{1}$ is given as the second component of the output polar vector calculated by adding and subtracting the products of a ray transfer matrix chain representing the transfer from each optical component to the detector with its small displacement and tilt vector (Ochi et al., 2008).

$$
\begin{aligned}
& \Delta=\Delta_{1}+\Delta_{2} \\
& \Delta_{1}=\left[\begin{array}{ll}
1 & 0
\end{array}\right]\left[\sum_{i=0}^{N-1} \prod_{j=i+1}^{N} A_{j}\left(I-A_{i}\right) \delta_{i}+\delta_{N}\right]
\end{aligned}
$$

where $I$ is a $2 \times 2$ identify matrix, $A_{j}$ is the ray transfer matrix characterizing the $j$ th optical component, and $\delta_{i}$ is the error vector consisting displacement and tilt of the $i$ th optical component with respect to the optical axis. $N$ is the number of optical components including a disc and air gaps.

The effect of displacement in the axial direction of the component changes the optical path length and shifts the beams as follows (Koos, 2007):

$$
r_{N}=A_{N} \cdots A_{i+1}\left[\begin{array}{cc}
1 & -\Delta x_{i} \\
0 & 1
\end{array}\right] A_{i}\left[\begin{array}{cc}
1 & \Delta x_{i} \\
0 & 1
\end{array}\right] A_{i-1} \cdots A_{0} r_{0}
$$

where $\Delta x_{i}$ is an axial misalignment of the $i$ th optical component, $r_{0}$, and $r_{N}$ are ray vectors representing beam height from the optical axis and its angle to the optical axis at the emitting point of the laser diode and on the sensing area of the detector, respectively. This leads to

$$
\Delta_{2}=\left[\begin{array}{ll}
1 & 0
\end{array}\right] \sum_{i=0}^{N-1} \Delta x_{i} \prod_{j=i+1}^{N} A_{j}\left[\begin{array}{cc}
-c_{i} & a_{i}-d_{i} \\
0 & c_{i}
\end{array}\right] r_{i}
$$

where $r_{i}$ is the desired ray vector representing beam height from the optical axis and its angle to the optical axis at the emitting point of the $i$ th optical component, $a_{i}, d_{i}$, and $c_{i}$ are upper-left, lower-right, and lower-left components of the $i$ th ray transfer matrix, respectively. This implies that we should only consider the prism and mirror, on which the laser beams enter with non-zero incident angles.

Equations (3), (4), and (6) indicate that the position deviation of the beam spot on the detector have a linearity with respect to displacement and tilt of each optical component. This is why the method for arranging adhesive joints of each optical component can compensate optical misalignment.

\section{Calculation}

\subsection{Conditions}

To calculate the optical misalignment over time due to the effects of heat and humidity on the adhesive joints, linear viscoelastic structural calculations were performed under thermal loading by using Abaqus ${ }^{\circledR}$.

Modulus of viscoelastic materials depends on time, and the relaxation processes accelerates as temperature increases. The thermal effect also changes in the dimension of the adhesive joint due to outgassing, moisture absorption when coupled with the humidity effect, and the thermal expansion. Thus, dimensional change due to moisture absorption or outgassing shrinkage of the adhesive joint was included in a coefficient of its thermal expansion (Pipes et al., 1976), which varies over time.

Figure 5 plots temperature profiles for loading to adhesive joints and to the other components. The sequence for considering this dimensional change of the adhesive joints is as follows. First, increase temperature uniformly from 26 to $60{ }^{\circ} \mathrm{C}$ in 420 seconds and then immediately alter the coefficient of thermal expansion of adhesives to that of moisture 
expansion including outgassing shrinkage. Second, keep the temperature at $60{ }^{\circ} \mathrm{C}$ for the other components for a period of time, while gradually increasing the temperatures of adhesive joints by just $1{ }^{\circ} \mathrm{C}$ to $61{ }^{\circ} \mathrm{C}$. At the end of the period of time, set the coefficient of thermal expansion of adhesive joints to zero and set the whole pickup at a uniform temperature of $60{ }^{\circ} \mathrm{C}$ within a millisecond. Finally, revert the coefficient of thermal expansion of adhesive joints to their original values and decrease the temperature to $26^{\circ} \mathrm{C}$ in 420 seconds.

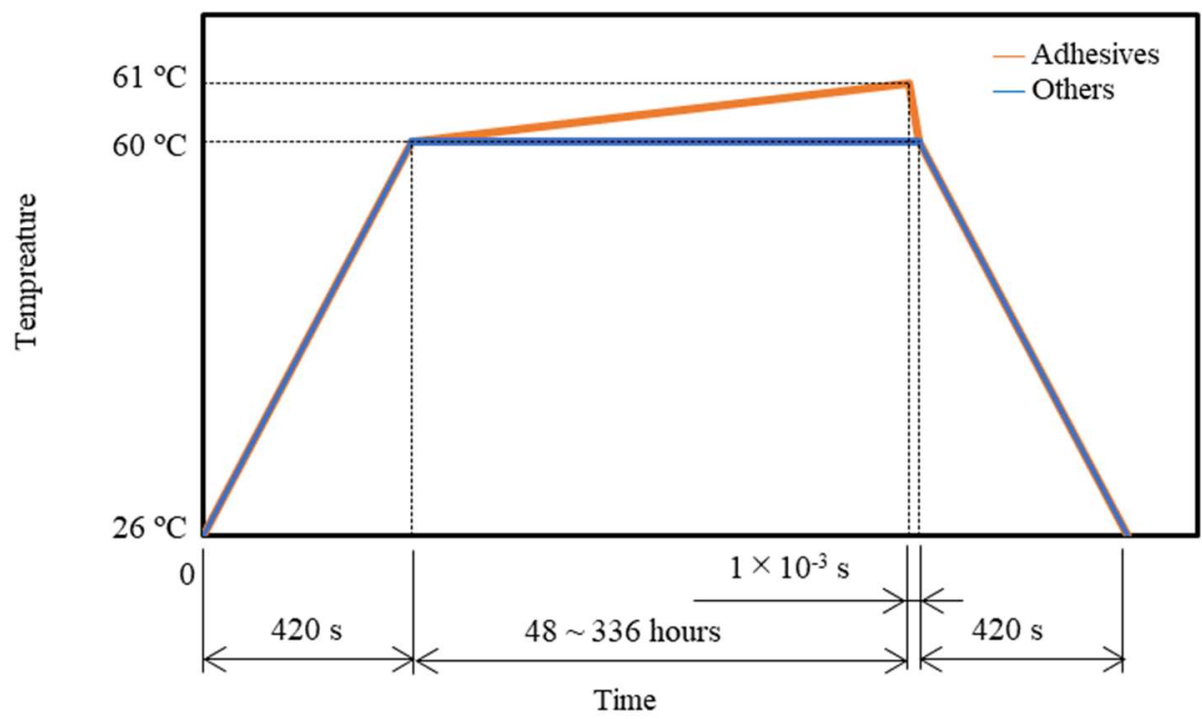

Fig. 5 Temperature profiles. Temperature of adhesive joints varies between 26 and $61{ }^{\circ} \mathrm{C}$ and that of the other components varies between $26^{\circ} \mathrm{C}$ and $60{ }^{\circ} \mathrm{C}$. Note that room temperature is assumed to be $26^{\circ} \mathrm{C}$ and that the thin optical pickup with small heat capacity is assumed to take 420 seconds to reach steady state temperature in a convection chamber.

\subsection{Viscoelasticity of adhesive}

\subsubsection{Measurement procedure}

To determine the viscoelastic properties of the adhesives, dynamic mechanical analysis tests were performed. Storage modulus $E^{\prime}$, loss modulus $E^{\prime \prime}$, and glass transition temperature $T_{g}$ of adhesive were measured in the tensile mode at frequencies of $0.628,1.88,6.28,18.8$, and $62.8 \mathrm{rad} / \mathrm{s}$ in a temperature range from -50 to $150{ }^{\circ} \mathrm{C}$ with a heating rate of $2{ }^{\circ} \mathrm{C} / \mathrm{min}$.

The time-temperature shift factor $a_{T}$ was fitted by using an Arrhenius plot, as shown in equation (7), by a reference temperature $T_{0}$ close to the $T_{g}$ and activation energy $\Delta H$, in order to cover a wider temperature range than the WilliamsLandel-Ferry equation, which has an applicable temperature range from $T_{g}$ to $T_{g}+100$ (Williams et al., 1955).

$$
\log a_{T}=0.434 \frac{\Delta H}{R}\left(\frac{1}{T}-\frac{1}{T_{0}}\right)
$$

where $R$ is the gas constant equal to $8.314 \mathrm{~J} / \mathrm{mol} / \mathrm{K}$ and $T$ is a temperature in Kelvins.

A master curve of the relaxation modulus was defined by using the generalized Maxwell model and specified as a set of Prony series, i.e.,

$$
E_{r}(\tau)=E_{g}-\sum_{k=1}^{M}\left(1-e^{-\frac{\tau}{\tau_{k}}}\right) E_{k}
$$

where $E_{g}$ is the instantaneous modulus, $E_{k}$ and $\tau_{k}$ are modulus and relaxation time of the $k$ th generalized Maxwell element, $\tau$ is time at $T_{0}$, and $M$ is the number of generalized Maxwell elements. 
The Prony series constants can be determined from the following implicit equations:

$$
\begin{aligned}
& E^{\prime}(\omega)=E_{g}-\sum_{k=1}^{M} \frac{1}{1+\omega^{2} \tau_{k}{ }^{2}} E_{k} \\
& E^{\prime \prime}(\omega)=\sum_{k=1}^{M} \frac{\omega \tau_{k}}{1+\omega^{2} \tau_{k}^{2}} E_{k}
\end{aligned}
$$

where $\omega$ and $\tau$ are angular frequency and time at $T_{0}$, respectively.

Note that the bulk modulus was assumed to be constant with temperature and time.

\subsubsection{Measurement results}

Obtained relaxation modulus curves for seven kinds of adhesives at $60{ }^{\circ} \mathrm{C}$ and their shift factors are shown in Figure 6(a) and (b), respectively. All adhesives were heated through their glass transition ranges during exposure at $60{ }^{\circ} \mathrm{C}$. The lower the glass transition temperature (the point where the logarithmic shift factor is equal to 1), the less residual stress occurred after the optical pickup was left at high temperature. The measurement temperature range selected were insufficient for adhesives $\mathrm{E}$ and $\mathrm{G}$. The relaxation modulus of them are supposed to be remain constant for duration times exceeding their last values shown in fig. 6(a).

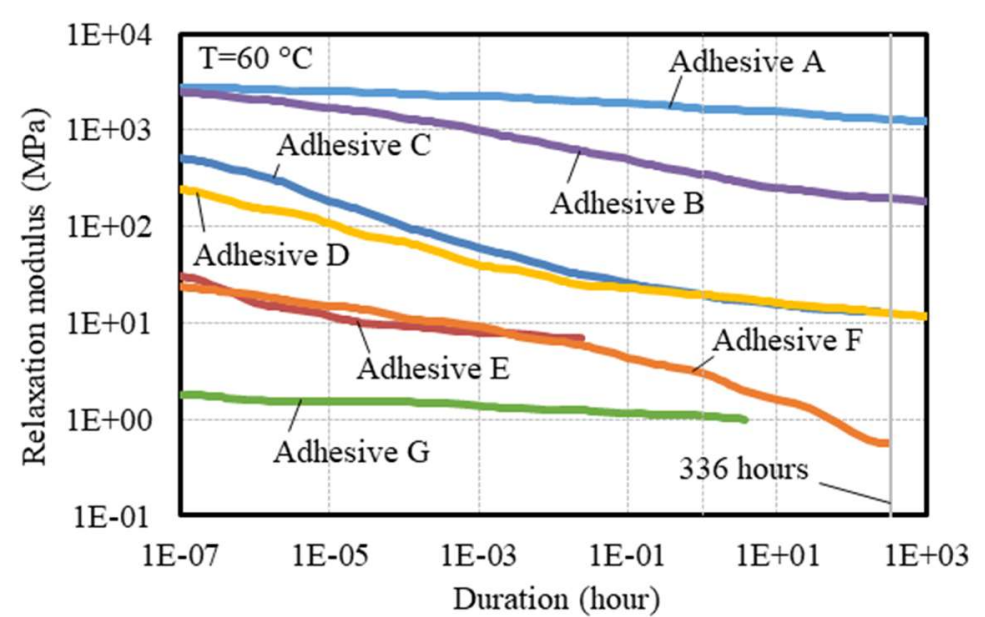

(a) Relaxation modulus

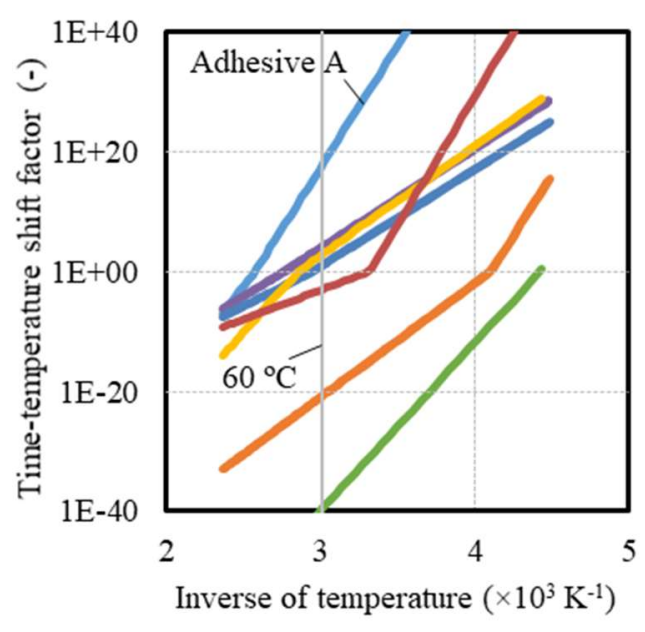

(b) Shift factor

Fig. 6 Thirty-term Prony series representing relaxation modulus for seven kinds of adhesives at $60{ }^{\circ} \mathrm{C}$ by shifting the curves measured at different frequencies along the horizontal axis. Shift factor with temperature is also shown in (b). The same color coding as in Figure 3 is used.

\subsection{Dimensional change due to moisture absorption and outgassing shrinkage 4.3.1 Measurement procedure}

To determine strain variation as a function of time after the adhesives were exposed to $60{ }^{\circ} \mathrm{C}$ with $90 \% \mathrm{RH}$, two parameters were measured: mass variation rate as a function of time and ratio of strain variation to the mass variation rate, called the coefficient of moisture expansion $(C M E)$.

Mass variation rate $c$ is expressed in mass percentage.

$$
c=\frac{\Delta m}{m_{0}} \times 100
$$

where $m_{0}$ is initial mass and $\Delta m$ is mass variation. 
The mass gain of the test piece adhesives corresponds to the amount of moisture absorbed whereas mass loss corresponds to outgassing. After being cured and weighted, test pieces were placed in a chamber at $60{ }^{\circ} \mathrm{C}$ and $90 \% \mathrm{RH}$ for 504 hours. During that exposure, the test pieces were periodically removed from the chamber, weighted, and returned. The mass variation was measured with an electronic balance.

$C M E$ is defined by the ratio of strain variation to the mass variation rate and is expressed as the following equation.

$$
C M E=\frac{\Delta l / l_{0}}{\Delta m / m_{0}}
$$

where $l_{0}$ is the initial length and $\Delta l$ is length variation.

The strain variation was approximated by using a linear function of mass variation rate, assuming that the adhesive uniformly expands or shrinks in all directions and that the density of the outgassed substance and moisture is 1 .

$$
\frac{\Delta l}{l_{0}}=\frac{\rho c}{300}
$$

where $\rho$ is density of the adhesive.

Strain variation was measured from the change in length between two points marked on test pieces. Test pieces were first preconditioned on a plate floating on water at $60{ }^{\circ} \mathrm{C}$ in a sealed box for a week, and their lengths and masses were measured. Their shrinkage and mass loss were then periodically measured while they were dried in a chamber at $60{ }^{\circ} \mathrm{C}$. The shrinkage was measured with a microscope. The mass loss measurements were the same as those for mass variation measurements.

\subsubsection{Measurement results}

Figure 7 summarizes the measured mass variation data. Adhesive A absorbed moisture quickly and was not saturated in 504 hours, whereas almost all other test pieces lost mass (outgassing). A possible explanation may be that the test pieces already contained moisture before curing. The second possibility is that UV-curing adhesives (Adhesives B to F) were not heated before measurement since they were cured at room temperature.

The measured shrinkage of adhesives relative to the mass loss is shown in Figure 8, together with corresponding linear approximations. Linear relationships between measured shrinkage and mass loss were basically observed. These results indicate that humidity greatly impacted the expansion of Adhesive A after it was left at high temperature. We assumed that moisture expansion would decrease residual stress in tension after the optical pickup was left at high temperature, whereas outgassing shrinkage increased instead.

Any other necessary properties for adhesives are shown in table 1.

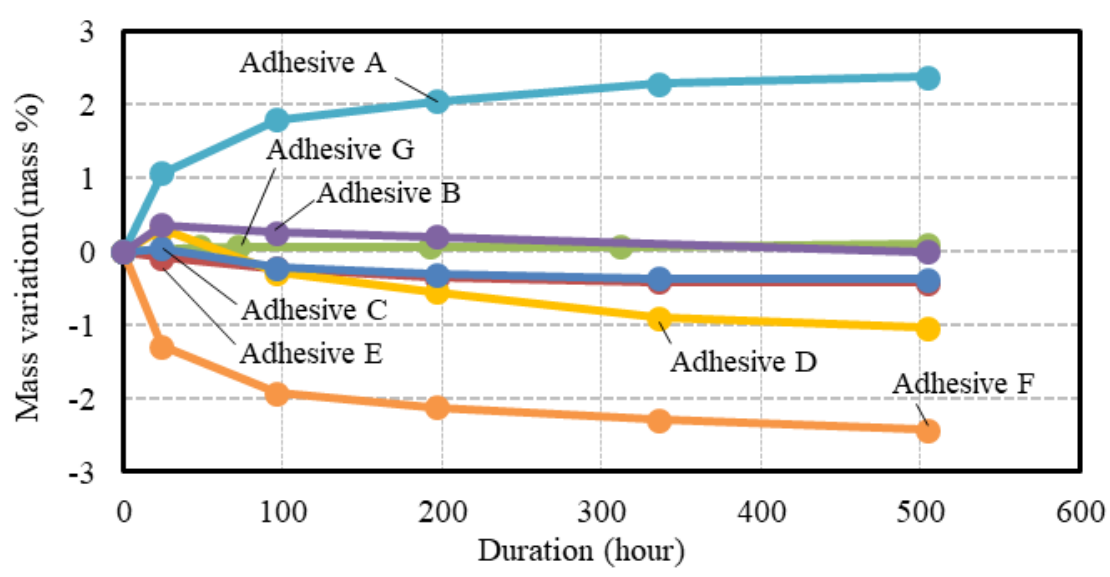

Fig. 7 Measured mass variation for seven kinds of adhesives left at $60{ }^{\circ} \mathrm{C}$ with $90 \%$ RH for 504 hours. The same color coding is used as in Figure 3. 


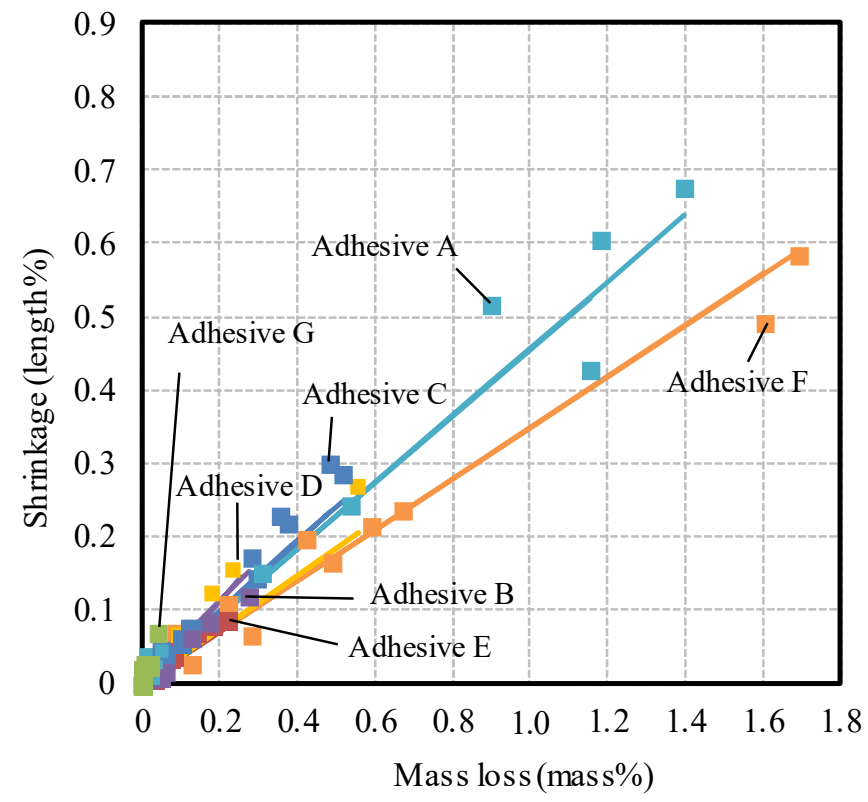

Fig. 8 Relationship between mass loss and shrinkage for seven kinds of adhesives. Linear approximations based on equation (13) are also shown. The same color coding is used as in Figure 3. The gradient of line is equal to CME.

Table 1 Thermal expansion coefficients and Poisson's ratios for adhesives.

\begin{tabular}{|c|c|c|}
\hline Adhesive & Poisson's ratio & Thermal expansion coefficient $\left(10^{-6} /{ }^{\circ} \mathrm{C}\right)$ \\
\hline A & 0.39 & 76 \\
\hline $\mathrm{B}$ & 0.45 & 85 \\
\hline $\mathrm{C}$ & 0.45 & 151 \\
\hline $\mathrm{D}$ & 0.45 & 192 \\
\hline $\mathrm{E}$ & 0.45 & 215 \\
\hline $\mathrm{F}$ & 0.45 & 282 \\
\hline $\mathrm{G}$ & 0.45 & 47 \\
\hline
\end{tabular}

\subsection{Material properties}

The main material properties used in this calculation are listed in table 2. Besides the adhesives, the optical pickup includes some optical and structural components made of polymer resin such as liquid-crystalline polymer, polyolefin, and glass epoxy. In this paper, the components are modeled as elastic bodies without moisture absorption and outgassing shrinkage, under the assumption that these resins have heat resistant and dimensional stability regardless of humid and dry conditions (Kawai, 1995; Tanaka and Onodera, 1999; Chikagawa, 1997; Okamoto, 2008; MITSUI CHEMICALS, INC, 2015; ZEON CORPORATION, 2018). Besides, anisotropic characteristic of liquid crystalline polymer, which is material for the fixed part in the actuator, is ignored, since the first concerns the comparison of two types of bonding structures for the laser diode. 
Table 2 Material properties for components.

\begin{tabular}{|c|c|c|c|c|}
\hline Component & Main material & $\begin{array}{l}\text { Young's } \\
\text { modulus } \\
(\mathrm{GPa})\end{array}$ & $\begin{array}{l}\text { Poisson's } \\
\quad \text { ratio }\end{array}$ & $\begin{array}{l}\text { Thermal } \\
\text { expansion } \\
\text { coefficient } \\
\left(10^{-6} /{ }^{\circ} \mathrm{C}\right)\end{array}$ \\
\hline Housing & Die-cast magnesium alloy & 45.7 & 0.3 & 26 \\
\hline Laser diode holders & Die-cast zinc alloy & $\begin{array}{l}89.2 \text { at } 26^{\circ} \mathrm{C} \\
75.9 \text { at } 60^{\circ} \mathrm{C}\end{array}$ & 0.3 & 27 \\
\hline Photo detector holders & Aluminum alloy & 96 & 0.3 & 27 \\
\hline Yoke and metal plates & Steel & 210 & 0.3 & 12 \\
\hline Magnets & Neodymium alloy & 160 & 0.33 & 12 \\
\hline $\begin{array}{l}\text { Fixed and moving parts in } \\
\text { lens actuators }\end{array}$ & Liquid crystal polymer & $\begin{array}{l}11 \text { at } 26^{\circ} \mathrm{C} \\
8.5 \text { at } 60^{\circ} \mathrm{C}\end{array}$ & 0.3 & $\begin{array}{r}29.9 \text { at most } 30^{\circ} \mathrm{C} \\
30.7 \text { at } 40^{\circ} \mathrm{C} \\
31.8 \text { at } 50{ }^{\circ} \mathrm{C} \\
33.6 \text { at } 60{ }^{\circ} \mathrm{C}\end{array}$ \\
\hline Detectors & Glass epoxy & 24.3 & 0.39 & 98 \\
\hline DVD/CD laser diode & Copper alloy & 125 & 0.38 & 17.5 \\
\hline BD laser diode & Steel & 210 & 0.3 & 12 \\
\hline \multirow{2}{*}{ Optical components } & Grass & 72 & 0.23 & 9.4 \\
\hline & Polyolefin & 2.4 & 0.34 & 74 \\
\hline
\end{tabular}

\section{Results}

Figure 9 compares position deviation of the beam spot on the detector after optical pickups with bottom- and topbonded laser diodes were left at $60^{\circ} \mathrm{C}$ with $90 \% \mathrm{RH}$ for $48,96,168$, and 336 hours.

The calculated position deviation (filled circles) vertically shifted from the measured values (empty circles) and the calculated difference between the two cases is about 1.5 times larger than the measured one. This might be attributed to the calculation ignoring the effects of cure shrinkage and moisture content before curing. Another likely explanation is lack of consideration for anisotropic characteristics of liquid crystalline polymer. The calculated values were still not very far from measurement variation, and the calculated results followed the growth of deviation when the laser diode was bonded to the bottom of the holder (top-bonded).

The calculated position deviations in the horizontal direction typically stayed within about $\pm 5 \%$ after 48 hours regardless of the bonding direction of the laser diode. In the vertical direction, the position deviation when the laser diode was bonded to the top of the holder (blue) remained as low as $\pm 5 \%$, whereas the position deviation when the laser diode was bonded to the bottom of the holder (red) grew steadily up to $-21.9 \%$ after the optical pickup was left at $60^{\circ} \mathrm{C}$ with $90 \% \mathrm{RH}$ for 336 hours. These calculation results are qualitatively consistent with measurement results, where a bottombonded laser diode is much better than the top-bonded laser diode. These results may indicate that a change in just the bonding direction of the laser diode could compensate for the optical misalignment due to long-term exposure to hot and humid conditions.

Figure 10 displays calculated $\mathrm{Z}$-axis displacement distributions of the optical assembly and housing left at $60^{\circ} \mathrm{C}$ with $90 \% \mathrm{RH}$ for 336 hours. In both cases, every optical component displaces and tilts as the housing becomes concave upward along both $\mathrm{X}$ and $\mathrm{Y}$ axes, except for the laser diode. They look very alike. This means the change in bonding direction of the laser diode barely affects the deformation of the housing. On the other hand, the laser diodes displaced in opposite directions due to the moisture expansion of Adhesive A. The bottom-bonded laser diode was lifted up whereas the topbonded laser diode was lifted down from the mounting surface in the holder. 
To support the effect of this change in bonding direction of the laser diodes, Figure 11 shows the details of how much the calculated displacement or tilt of each optical component contributes to the position deviation of the beam spot on the detector in the vertical direction. These results suggest that the position deviation in the vertical direction is mainly caused by the Z-displacement of the laser diode, pitching of the prism, and the Z-displacement of the detector. In both cases in Figure 11, the contributions of the Z-displacement of the detector and the pitching of the prism were almost constant, but the Z-displacement of the laser diode had the opposite effect depending on its bonding direction. The Zdisplacement of the bottom-bonded laser diode deviated beam spot upward with Z-displacement of the detector, but at the same time pitching of the prism balanced it, so the beam spot remained stable. In contrast, the Z-displacement of the top-bonded laser diode deviated the beam spot downward with the pitching of the prism three times as much as the Zdisplacement of the detector deviated the beam spot upward, resulting in position deviation. The results support the effectiveness of the change in bonding direction of the laser diode. Our method of arranging adhesive joints also appeared to be well suited to passively compensate for the permanent optical misalignment due to long-term exposure to hot and humid conditions.

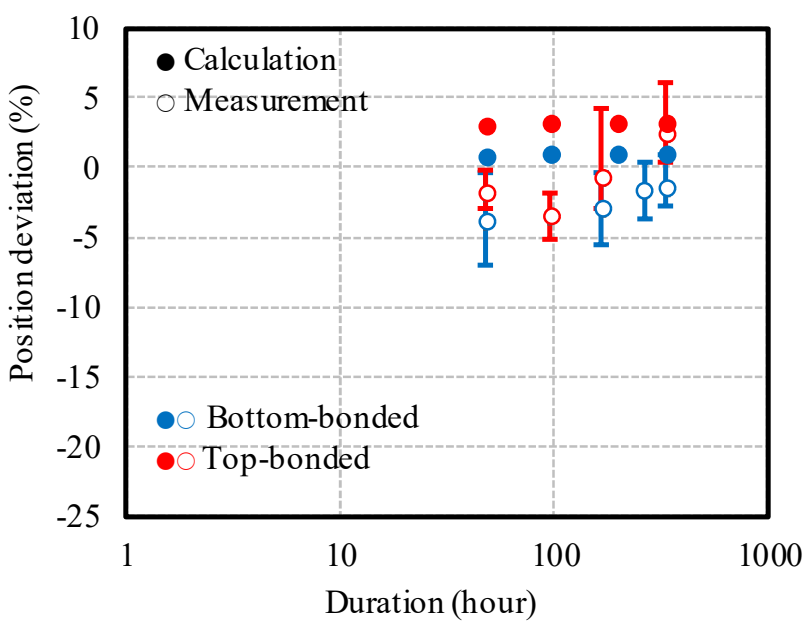

(a) Horizontal direction

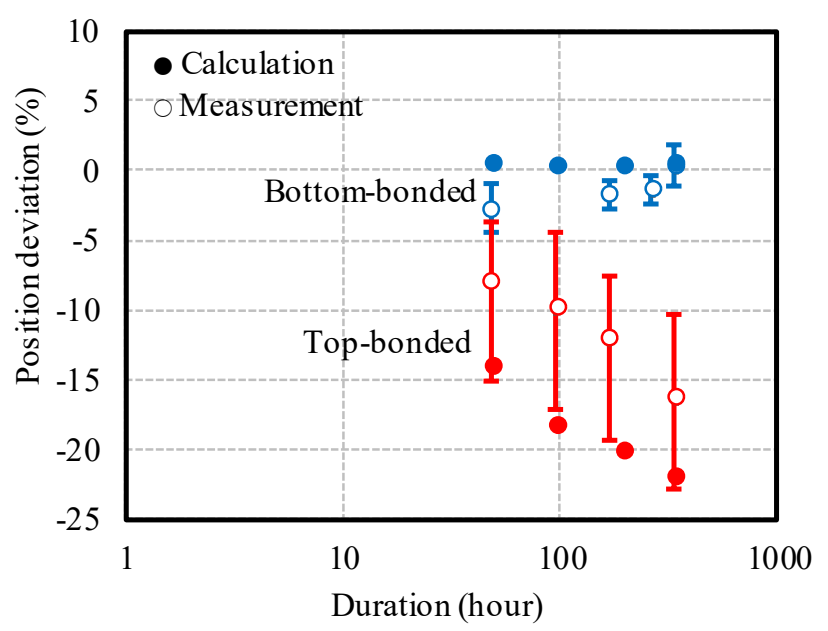

(b) Vertical direction

Fig. 9 Comparison in position deviation of beam spot on detector of optical pickups with bottom- and top-bonded laser diodes left at $60^{\circ} \mathrm{C}$ with $90 \% \mathrm{RH}$ for $48,96,168$, and 336 hours. Filled circles are calculated results and empty circles are measured means with range bars that represent minimum and maximum values of measured deviation. They are color-coded by bonding direction of laser diode: Red points represent results with bottom-bonded laser diode, and blue ones represent results with top-bonded laser diode. Note that the former case was measured by using four prototypes and the latter by using ten prototypes.

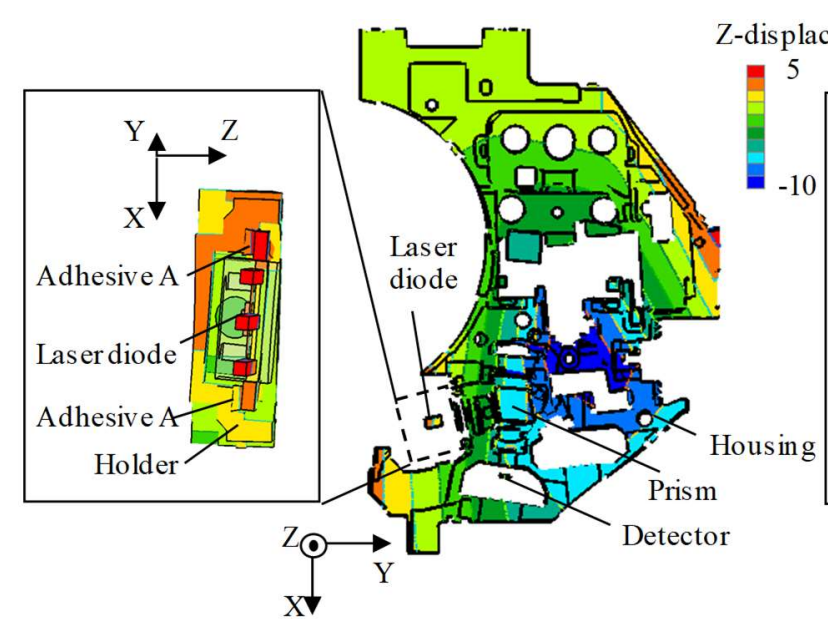

(a) Bottom-bonded

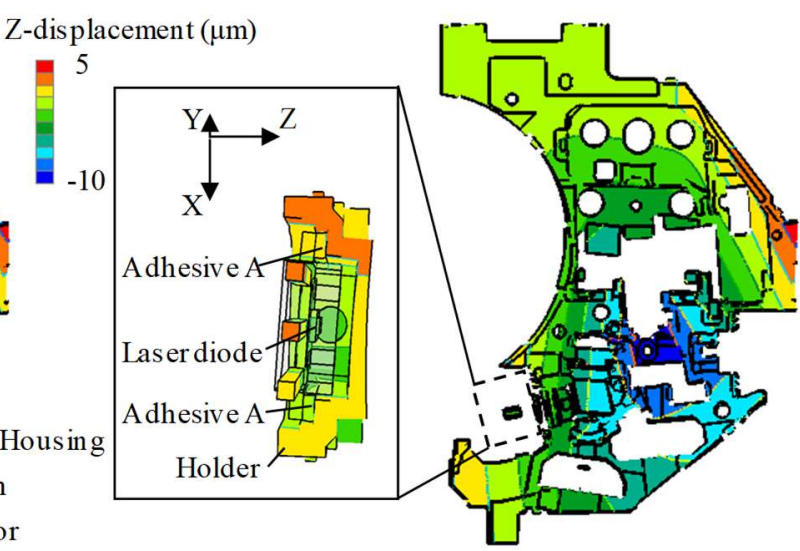

(b) Top-bonded

Fig. 10 Calculated Z-axis displacement distribution of optical assembly and housing left at $60{ }^{\circ} \mathrm{C}$ with $90 \% \mathrm{RH}$ for 336 hours. In both cases, all optical components except laser diode displace and tilt as housing deforms. 


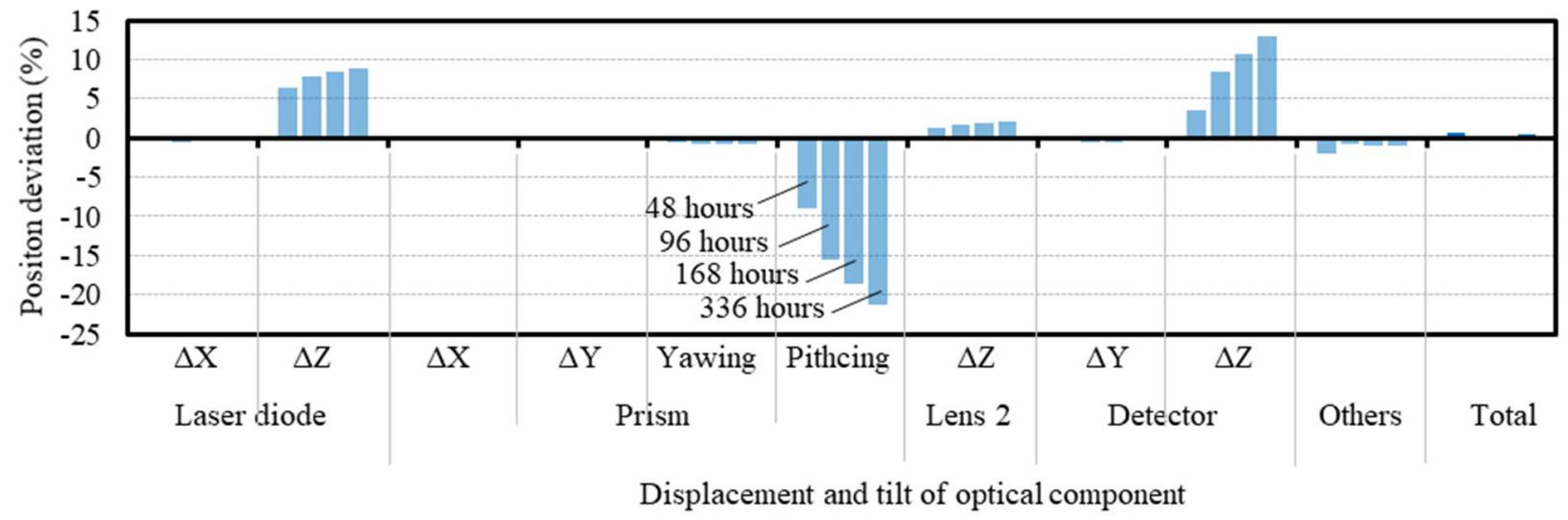

(a) Bottom-bonded

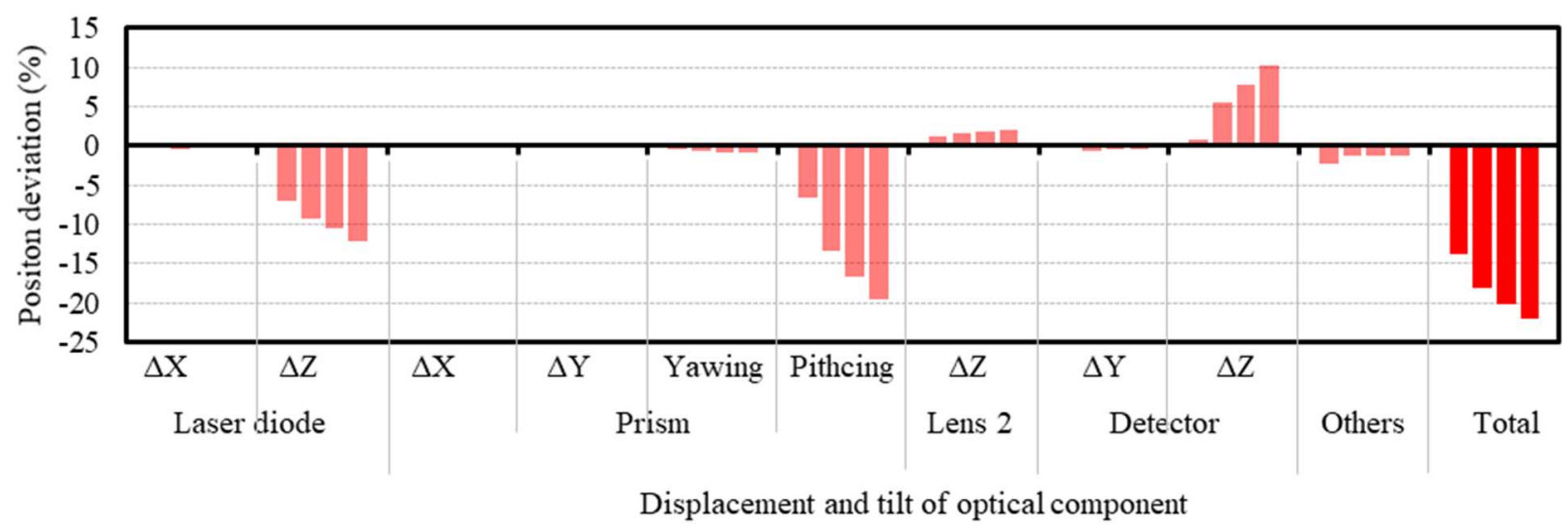

(b) Top-bonded

Fig. 11 Breakdown of calculated position deviation in vertical direction after optical pickups with bottom- and top-bonded laser diode were left at $60^{\circ} \mathrm{C}$ with $90 \% \mathrm{RH}$ for $48,96,168$, and 336 hours in terms of contributing displacement and tilt of each optical component. The values on the far right (thick) were identical to calculated results in Figure 8. (a) and (b) show similar but different effects of $\Delta \mathrm{Z}$ of laser diode.

\section{Conclusion}

By calculation and experiment, we compared the beam spot deviations on a detector, which is an indicator of misalignment of optical assembly, after an optical pickup was left at $60{ }^{\circ} \mathrm{C}$ and $90 \% \mathrm{RH}$ between two types of adhesive joints of a laser diode. The results are summarized as follows. The calculated and measured results show similar trends, though the calculated position deviation vertically shifted from the measured values. The position deviations in the horizontal direction typically stayed within about $\pm 5 \%$ after 48 hours. In the vertical direction, position deviation was correlated with the bonding direction of the laser diode. The position deviation when the laser diode was bonded to the top of the holder (bottom-bonded) remained as low as $\pm 5 \%$, whereas the position deviation when the laser diode was bonded to the bottom of the holder (top-bonded) grew steadily up to $-21.9 \%$ after the optical pickup was left at $60{ }^{\circ} \mathrm{C}$ with $90 \% \mathrm{RH}$ for 336 hours. The calculation results supported the reason for this difference being that upward displacement of a bottom-bonded laser diode could compensate for optical misalignment caused by tilt of the prism and displacement of the detector along the warp deformation of the housing.

Our method of arranging adhesive joints for an optical assembly was proved to effectively enhance long-term as well as short-term optical alignment stabilities without the use of extra active control. We strongly believe that this method is useful for assembling optical devices because it gives a detailed knowledge of how adhesive joints influence on the optical alignment and suggests how to avoid possible dangerous adhesive joint arrangements. 


\section{References}

Chikagawa, M., Liquid crystalline polymer in electronic components, Journal of SHM (in Japanese), Vol. 13, No. 6 (1997), pp.16-20.

Chikuma, K., Basics of optical pick-up and its packaging technology, Journal of Japan Institute of Electronics Packaging (in Japanese), Vol. 1, No. 3 (1998), pp.230-234.

Iida, T., Trouble-shooting of UV adhesives for opto-electronics, Journal of The Adhesion Society of Japan (in Japanese), Vol. 43, No.2 (2007), pp.72-78.

Iwai, M., Optical pickups for mobile multi-media, Japanese Journal of Optics (in Japanese), Vol. 30, No. 6 (2001), pp. 376-380.

Kato, T., Miyamori, J., Satomi, K., Saito, Y., and Ishida, N., Low cost CD pickup for car use (P9), PIONEER R\&D (in Japanese), Vol. 11, No. 3 (2001), pp.42-47.

Kawai, H., Plastic molding materials for precision optics, Japanese Journal of Optics (in Japanese), Vol. 24, No. 2 (1995), pp.69-75.

Koos, G., Matrix methods for optical layout (2007), p.56, SPIE.

Manoh, K., Yoshida, H., Kobayashi, T., Takase, M., Yamauchi, K., Fujiwara, S., Ohno, T., Nishi, N., Ozawa, M., Ikeda, M., Tojyo, T., and Taniguchi, T., Small integrated optical head device using a blue-violet laser diode for Blu-ray Disc system, Japanese Journal of Applied Physics, Vol. 42, No. 2B (2003), pp.880-884.

Matsuo, K., Effect of shrinkage of adhesive resin for optical elements, The Japan Society for Precision Engineering (in Japanese), Vol. 64, No. 2 (1998), pp.202-205.

MITSUI CHEMICALS, INC. (2015), APEL ${ }^{\mathrm{TM}}$ (online), available from $<$ https://www.mitsuichem.com/sites/default/files/media/document/2018/apel_j.pdf>, (accessed on 18 June, 2019).

Miyauchi T., Shimoyama S., Sekiya S., LD/PD precision inspection machines contributing to improvement in quality of DVD optical pickups, TOSHIBA REVIEW (in Japanese), Vol. 58, No. 12 (2003), pp. 58-61.

Nakai, K., Ohmaki, M., and Takeshita, N., High-accuracy focus error detection by offset correction in optical pickup, Proceedings of ITE Annual Convention 2013 (in Japanese) (2013).

Nemoto, K. and Honda, K., Integrated optical devices for optical disk applications, IEICE Transactions on Electronics, Vol. E85-C, No. 4 (2002), pp.1001-1008.

Ochi, M., Ide, T., Izumi, K., Watanabe, M., and Satake, M., Design of highly reliable optical pickup, Proceedings of the 16th International Symposium on Optical Memory (2006), pp.150-151.

Ochi, M., Satake, M., Kawamura, T., Izumi, K., Ide, T. and Oozeki, Y., High-environmental resistance jisso structure for a slim optical head with a resin chassis, Transactions of the Japan Society of Mechanical Engineers, Series C (in Japanese), Vol. 74, No. 740 (2008), pp. 753-759.

Okamoto, S., Liquid Crystalline Polymer, Journal of The Society of Rubber Industry (in Japanese), Vol. 81, No. 3 (2008), pp.86-92.

Pipes, R. B., Vinson, J. R., and Chou, T. W., On the hygrothermal response f laminated composite systems, Journal of Composite Materials, Vol. 10 (1976), pp.129-148.

Prabhu, K. S., Schmitz, T. L., Ifju, P. G., and Daly. J. G., A Survey of Technical Literature on Adhesive Applications for Optics, Proceedings of SPIE, Vol. 6665 (2007).

Sakamoto, Y. and Saito, S., Light-curing adhesive for image sensors and optical pickups, ThreeBond Technical News, No. 84 (2014).

Tanaka, Y. and Onodera, M., Liquid crystal polymer material for LSI-mounting, Japan Institute of Electronics Packaging (in Japanese), Vol. 2 No. 2(1999), pp. 84-89.

Terashi T., Ohshima H., Taneda Y., and Kamada T., Adjustment and assembly technique for integrated device of optical pickup, Ricoh Technical Report (in Japanese), No.29 (2003), pp.53-57.

Williams, M. L., Landel, R. F., and Ferry, J. D., The temperature dependence of relaxation mechanisms in amorphous polymers and other glass-forming liquids, Journal of the American Chemical Society, Vol. 77, No. 14 (1955), pp.3701-3707.

Yanagihara, E., Adhesives for electronic assemblies, Journal of the Adhesion Society of Japan (in Japanese), Vol. 37 , No.10 (2001), pp.20-29.

Yoshida, S., Minami, K., Okada, K., Yamamoto, H., Ueyama, T., Sakai, K., and Kurata, Y., Optical pickup employing a 
Ochi, Kitada and Konishi, Journal of Advanced Mechanical Design, Systems, and Manufacturing, Vol.13, No.3 (2019)

hologram-laser-photodiode unit, Vol. 39, No. 2B (2000), pp. 877-882.

Yoshimoto, K., Ito, O., and Tanaka K., Compensation method of push-pull tracking servo offset of optical disk drives, Journal of the Institute of Television Engineers of Japan (in Japanese), Vol. 44, No. 10 (1990), pp.1398-1402.

ZEON CORPORATION (2018), ZEONEX® (online), available from <http://www.zeon.co.jp/content/200133136.pdf>, (accessed on 18 June, 2019). 